\title{
Nonthermal steady states after an interaction quench in the Falicov-Kimball model
}

\author{
Martin Eckstein and Marcus Kollar \\ Theoretical Physics III, Center for Electronic Correlations and Magnetism, \\ Institute for Physics, University of Augsburg, 86135 Augsburg, Germany
}

(Dated: July 18, 2007)

\begin{abstract}
We present the exact solution of the Falicov-Kimball model after a sudden change of its interaction parameter using non-equilibrium dynamical mean-field theory. For different interaction quenches between the homogeneous metallic and insulating phases the system relaxes to a non-thermal steady state on time scales on the order of $\hbar$ /bandwidth, showing collapse and revival with an approximate period of $h$ /interaction if the interaction is large. We discuss the reasons for this behavior and provide a statistical description of the final steady state by means of generalized Gibbs ensembles.
\end{abstract}

PACS numbers: 03.75.Ss, 05.30.Fk, 71.27.+a

How does an isolated quantum-mechanical many-body system develop after it is suddenly forced out of thermal equilibrium? Under which conditions does it relax to a new steady state, and how fast? Is it ergodic so that it reaches a new thermodynamic equilibrium, or does the final state depend on the initial state? Recently it has become feasible to study these fundamental questions experimentally and theoretically. In experiments with ultracold atomic gases [1] it is possible to subject a prepared initial state to a rapid change of system parameters. Long observation times are possible due to the excellent isolation from the environment. For example, Bose-Einstein condensates (BECs) were quenched across the superfluid-insulator transition and back [2], their collapse and revival after a quench was observed [3], a quenched spinor BEC was found to exhibit spontaneous symmetry breaking [4], and a quantum version of Newton's cradle was found not to thermalize [5].

One might expect that a quenched system with many interacting degrees of freedom will relax to a new thermal state, characterized only by a few thermodynamic variables such as internal energy and particle number. However this may not be the case if the system is integrable, because then the final state is constrained by infinitely many constants of motion. Indeed, theoretical studies for one-dimensional hard-core bosons [6, 7] (experimentally realized in Ref. [5) and for the Luttinger model [8] found that these integrable systems relax to non-thermal steady states. Nevertheless for both models the final state is described by a generalized Gibbs ensemble [6], which maximizes the entropy subject to all constraints. On the other hand, for non-integrable and unconstrained systems the usual Gibbs ensemble should describe the final steady state. In contrast to this expectation recent numerical studies for finite one-dimensional systems of soft-core bosons [9] and spinless fermions [10] did not find thermalization. While the reasons for this behavior are not yet understood, hard-core bosons in two dimensions do thermalize as expected [11]. Clearly finitesize effects must be well-controlled in all such calculations in order to obtain the correct behavior at large times.

Dynamical mean-field theory (DMFT) [12, 13], which has become a standard technique for correlated systems in equilibrium, can also provide insight into their quantum dynamics, e.g., in the presence of time-dependent external fields 14, 15]. DMFT has the conceptual advantages that it is formulated in the thermodynamic limit so that finite-size lattice effects are eliminated, and that it becomes exact for high-dimensional lattices. As such, it is complementary to numerical methods for finite low-dimensional systems. The characteristic features of DMFT for fermions [13] or bosons [16], namely a local self-energy derived from a local action with selfconsistency condition, persist also for non-equilibrium situations.

In this paper we use DMFT to study quenches in the interaction parameter of the Falicov-Kimball (FK) model. This lattice model describes itinerant $c$ electrons and immobile $f$ electrons that interact via a repulsive local interaction $U$ [17]. The Hamiltonian is given by

$$
H=\sum_{i j} V_{i j} c_{i}^{\dagger} c_{j}+E_{f} \sum_{i} f_{i}^{\dagger} f_{i}+U \sum_{i} f_{i}^{\dagger} f_{i} c_{i}^{\dagger} c_{i},
$$

i.e., it is similar to the Hubbard model except that only one electron species can hop between lattice sites. In DMFT the effective local action for the $c$ particles is quadratic, so that their Green function can be obtained exactly [18, 19]. The equilibrium solution describes correlation-induced transitions between metallic, insulating, and charge-ordered phases [20]. The FK model proved very useful as a guide for the application of DMFT to the Hubbard model. It currently plays a similar role for nonequilibrium DMFT, in particular since no appropriate real-time impurity solver is yet available for the Hubbard model, although, e.g., time-dependent numerical-renormalization group [21] is a promising candidate. So far, however, even the selfconsistency equation has required tremendous numerical effort for a general nonequilibrium situation due to lack of time-translational invariance [15]. For the investigation of an interaction quench we consider a semi-elliptical density of states, which leads to a dramatic simplification of the self-consistency equation both for the FK and Hubbard model. 
We assume that the system is prepared in thermal equilibrium at temperature $T$ for times $t<0$; at $t=0$ the interaction is suddenly switched from the value $U_{-}$to a new value $U_{+}$, so that the time evolution for $t \geq 0$ is governed by the new Hamiltonian 22]. Below we obtain the exact non-equilibrium Green function for arbitrary quenches and arbitrary large times.

Non-equilibrium DMFT. - The theory is formulated in terms of contour-ordered real-time Green functions. In general, this formalism is appropriate to describe an isolated system, where the initial state is a density matrix [23]. We use the Keldysh Green functions $G_{i j}\left(t, t^{\prime}\right)=$ $-i\left\langle\mathrm{~T}_{\mathcal{C}} c_{i}(t) c_{j}^{\dagger}\left(t^{\prime}\right)\right\rangle$, which are defined on the contour $\mathcal{C}$ that runs from a negative $t_{\min }$ to a positive $t_{\max }$, then from $t_{\max }$ to $t_{\min }$, and finally to $t_{\min }-i \beta[15]$. Here $\langle\cdot\rangle=$ $\operatorname{Tr}\left[e^{-\left(H\left(t_{m i n}\right)-\mu N\right) / T}.\right]$ is the thermal expectation value with chemical potential $\mu$ and total particle number $N$. For the FK model the local Green function $G\left(t, t^{\prime}\right)$ in the homogeneous phase is calculated from a local action 15, 18],

$$
\begin{aligned}
G\left(t, t^{\prime}\right) & =-i \frac{\operatorname{Tr}_{c, f}\left[e^{-\beta H_{0}} \mathrm{~T}_{\mathcal{C}} S_{1} S_{2} c(t) c^{\dagger}\left(t^{\prime}\right)\right]}{\operatorname{Tr}_{c, f}\left[e^{-\beta H_{0}} \mathrm{~T}_{\mathcal{C}} S_{1} S_{2}\right]} \\
S_{1} & =\exp \left(-i \int_{\mathcal{C}} d \bar{t} \int_{\mathcal{C}} d \bar{t}^{\prime} c^{\dagger}(\bar{t}) \Lambda\left(\bar{t}, \bar{t}^{\prime}\right) c\left(\bar{t}^{\prime}\right)\right) \\
S_{2} & =\exp \left(-i \int_{\mathcal{C}} d \bar{t} U(\bar{t}) c^{\dagger}(\bar{t}) c(\bar{t}) f^{\dagger}(\bar{t}) f(\bar{t})\right),
\end{aligned}
$$

where the operators are in the interaction representation with respect to $H_{0}=\left(E_{f}-\mu\right) f^{\dagger} f-\mu c^{\dagger} c$, and $\hbar=1$. After tracing out the $f$ electrons and setting $w_{1}=\left\langle f^{\dagger} f\right\rangle$ $=1-w_{0}$ one has

$$
G\left(t, t^{\prime}\right)=w_{0} Q\left(t, t^{\prime}\right)+w_{1} R\left(t, t^{\prime}\right),
$$

where $Q\left(t, t^{\prime}\right)$ and $R\left(t, t^{\prime}\right)$ are given by (2) but without $\operatorname{Tr}_{f}$ and with $f^{\dagger}(\bar{t}) f(\bar{t})$ replaced by 0 and 1 , respectively. From (2) follow the equations of motion

$$
\begin{aligned}
{\left[i \partial_{t}^{\mathcal{C}}+\mu\right] Q\left(t, t^{\prime}\right)-(\Lambda * Q)\left(t, t^{\prime}\right) } & =\delta^{\mathcal{C}}\left(t, t^{\prime}\right), \\
{\left[i \partial_{t}^{\mathcal{C}}+\mu-U(t)\right] R\left(t, t^{\prime}\right)-(\Lambda * R)\left(t, t^{\prime}\right) } & =\delta^{\mathcal{C}}\left(t, t^{\prime}\right),
\end{aligned}
$$

where $(f * g)\left(t, t^{\prime}\right)=\int_{\mathcal{C}} d \bar{t} f(t, \bar{t}) g\left(\bar{t}, t^{\prime}\right)$ denotes the convolution, $\partial_{t}^{\mathcal{C}}$ the derivative, and $\delta^{\mathcal{C}}\left(t, t^{\prime}\right)$ the delta function along the contour [15], and the Green functions obey antiperiodic boundary conditions.

In DMFT the contour self-energy is local and its skeleton expansion in terms of the contour Green function is the same as that of the self-energy of the local problem (2), determined from its Dyson equation

$$
\left(i \partial_{t}^{\mathcal{C}}+\mu\right) G\left(t, t^{\prime}\right)-([\Lambda+\Sigma] * G)\left(t, t^{\prime}\right)=\delta^{\mathcal{C}}\left(t, t^{\prime}\right) .
$$

On the other hand, the lattice Dyson equation provides a relation between the self-energy and the lattice contour Green function $G_{i j}\left(t, t^{\prime}\right)$,

$$
\left(i \partial_{t}^{\mathcal{C}}+\mu-\epsilon_{\boldsymbol{k}}\right) G_{\boldsymbol{k}}\left(t, t^{\prime}\right)-\left(\Sigma * G_{\boldsymbol{k}}\right)\left(t, t^{\prime}\right)=\delta^{\mathcal{C}}\left(t, t^{\prime}\right),
$$

where $\epsilon_{\boldsymbol{k}}$ are the eigenvalues of the matrix $V_{i j}$. In the corresponding eigenbasis the lattice contour Green function $G_{\boldsymbol{k}}\left(t, t^{\prime}\right) \equiv G_{\epsilon_{\boldsymbol{k}}}\left(t, t^{\prime}\right)$ is diagonal and depends on $\boldsymbol{k}$ only through $\epsilon_{\boldsymbol{k}}$. The self-consistency equation

$$
G\left(t, t^{\prime}\right)=\int d \boldsymbol{k} G_{\boldsymbol{k}}\left(t, t^{\prime}\right)=\int d \epsilon \rho(\epsilon) G_{\epsilon}\left(t, t^{\prime}\right),
$$

then closes the problem, i.e., there are three equations (3), (41), (5) for three unknowns $G\left(t, t^{\prime}\right), \Lambda\left(t, t^{\prime}\right), \Sigma\left(t, t^{\prime}\right)$. For a general density of states $\rho(\epsilon)$ the numerical evaluation of (5) is expensive, because the integral equation (5a) must be solved for every integration point in $5 \mathrm{~b}$ 15]. This problem simplifies dramatically for a semielliptic density of states $\rho(\epsilon)=\sqrt{4 V^{2}-\epsilon^{2}} / 2 \pi V$. In this case, the Hilbert transform $g(z)=\int d \epsilon \rho(\epsilon) /(z-\epsilon)$ satisfies the equation $z g=1+V^{2} g$, and this also holds for linear operators 24], e.g., $z=\left(i \partial_{t}^{\mathcal{C}}+\mu-\Sigma\right)$ and $g=$ $G\left(t, t^{\prime}\right)$. Thus (5) reduces to

$$
\left(i \partial_{t}^{\mathcal{C}}+\mu\right) G\left(t, t^{\prime}\right)-\left(\left[\Sigma+V^{2} G\right] * G\right)\left(t, t^{\prime}\right)=\delta^{\mathcal{C}}\left(t, t^{\prime}\right),
$$

so that, by comparison with (44),

$$
\Lambda\left(t, t^{\prime}\right)=V^{2} G\left(t, t^{\prime}\right) .
$$

Analytic solution. - We now solve (3) and (6) for an interaction quench at $t=0$. Because the Hamiltonian does not change for times $t<0$, the Green functions take their equilibrium values when both $t<0$ and $t^{\prime}$ $<0$. We take this as an initial condition in Eq. (3) and remove the vertical part of the contour by letting $t_{\min } \rightarrow-\infty$; correlations such as $G\left(t, t_{\min }-i \tau\right)$ between times $t$ on the real part of the contour and $t_{\min }-i \tau$ on the imaginary part vanish in this limit. Using Langreth rules 23] we then recast (3) into a set of coupled integrodifferential equations for the lesser component $G^{<}\left(t, t^{\prime}\right)$ $=i\left\langle c^{\dagger}\left(t^{\prime}\right) c(t)\right\rangle$ and the retarded component $G^{R}\left(t, t^{\prime}\right)=$ $-i \Theta\left(t-t^{\prime}\right)\left\langle\left\{c^{\dagger}\left(t^{\prime}\right), c(t)\right\}\right\rangle$. Directly from these rules and the fact that any retarded function $f\left(t, t^{\prime}\right)$ must vanish for $t<t^{\prime}$, one can see that within these equations the retarded Green functions with $t>t^{\prime}>0$ and $0>t>t^{\prime}$ are decoupled from all other components. Moreover, the corresponding two sets of equations differ only in the value of $U$, and both are translational invariant in time. Thus they can be written in terms of the Fourier transforms $g_{ \pm}^{R}(z)$ ( \pm for $t, t^{\prime} \gtrless 0$, respectively) with respect to $t-t^{\prime}$,

$$
\begin{aligned}
& g_{ \pm}^{R}(z)=w_{0} q_{ \pm}^{R}(z)+w_{1} r_{ \pm}^{R}(z), \\
& q_{ \pm}^{R}(z)=\left[z+\mu-V^{2} g_{ \pm}^{R}(z)\right]^{-1} \\
& r_{ \pm}^{R}(z)=\left[z+\mu-V^{2} g_{ \pm}^{R}(z)-U_{ \pm}\right]^{-1} .
\end{aligned}
$$

The same set of cubic equations determines the equilibrium Green function [19], but in the present case $\mu$ is always the chemical potential of the initial thermal state [22]. The remaining components of retarded and lesser Green functions are then solved for by using separate 
Fourier transform with respect to $t$ and $t^{\prime}$ in each region where both $t$ and $t^{\prime}$ do not change sign. For the most important sector with both time arguments after the quench, we obtain $G_{++}^{<}\left(t, t^{\prime}\right)=G^{<}\left(t, t^{\prime}\right) \Theta(t) \Theta\left(t^{\prime}\right)$ by double Fourier transform,

$$
\begin{aligned}
\tilde{G}_{++}^{<}(z, \eta) & =\int d t e^{i z t} \int d t^{\prime} e^{i \eta t^{\prime}} G_{++}^{<}\left(t, t^{\prime}\right) \\
& =-\int d \omega \frac{f(\omega)}{2 \pi V^{2}} \frac{M(z, \omega)+M\left(-\eta^{*}, \omega\right)^{*}}{z+\eta}
\end{aligned}
$$

with the abbreviations

$$
\begin{aligned}
M(z, \omega) & =\left[1-K^{A}(z, \omega)\right]^{-1}-\left[1-K^{R}(z, \omega)\right]^{-1}, \\
K^{\lambda}(z, \omega) & =V^{2}\left[w_{0} q_{+}^{R}(z) q_{-}^{\lambda}(\omega)+w_{1} r_{+}^{R}(z) r_{-}^{\lambda}(\omega)\right] .
\end{aligned}
$$

Note that the initial state enters (8b) via the Fermi function, $f(\omega)=1 /\left(1+e^{\beta \omega}\right)$. Similar expressions are derived for the other Green functions $Q^{<}$and $R^{<}$[24].

Time-dependent expectation values of observables are now obtained by inverse Fourier transformation and numerical integration. Below we discuss the double occupation $D(t)=-i w_{1} R^{<}(t, t)$ and the momentum distribution, i.e., the occupation $n(\epsilon, t)$ of single-particle eigenstates $|\epsilon\rangle$. The latter is given by $n(\epsilon, t)=-i G_{\epsilon}^{<}(t, t)$ as defined below (5a). The total density $n_{c}$ is conserved, and the internal energy $E=\langle H\rangle+\mu n_{c}$ jumps by $\Delta E=$ $\left(U_{+}-U_{-}\right) D\left(0^{-}\right)$at the quench [22].

Simplifications occur in the limit of infinite waiting time. For $t \rightarrow \infty$ the partial Fourier transformation $G^{<}(\omega, t)=\int d s e^{i \omega s} G^{<}(t+s / 2, t-s / 2)$ has a well-defined limit $g_{\infty}^{<}(\omega)$, which is determined only by the singularity at $z=-\eta$ in $8 \mathrm{~b}$. While $G^{<}(\omega, t)$ is complex in general, its long-time limit is purely imaginary,

$$
\begin{aligned}
g_{\infty}^{<}(\omega) & =\int d \omega^{\prime} \frac{f\left(\omega^{\prime}\right)}{\pi V^{2}} i \operatorname{Re}\left[M\left(\omega+i 0, \omega^{\prime}\right)\right] \\
& =2 \pi i h(\omega) A_{+}(\omega) .
\end{aligned}
$$

Plugging this result back into (3) and (6) we find that the steady state is characterized by (i) a real positive function $h(\omega)$ which replaces the Fermi function $f(\omega)$ in the equilibrium expressions, and (ii) the temperatureindependent spectrum for $U_{+}$as given by $A_{+}(\omega)=$ $\operatorname{Im}\left[g_{+}^{A}(\omega)\right] / \pi$. In particular, $E(t>0)=\int d \omega h(\omega)(\omega+\mu)$ $A_{+}(\omega), D_{\infty}=w_{1} \int d \omega h(\omega) \operatorname{Im}\left[r_{+}^{A}(\omega)\right] / \pi$, and $n_{\infty}(\epsilon)=$ $\int d \omega h(\omega) \operatorname{Im}\left[\left(\omega-i 0-\epsilon-\Sigma_{+}^{A}(\omega)\right)^{-1}\right] / \pi$. It is remarkable that subsequent quenches can be accounted for by simply replacing the initial occupation function $f(\omega)$ with the steady-state occupation function $h(\omega)$ in (8b) and (91).

Non-thermal steady state. - In the following we focus on the case of half-filling for both $c$ and $f$ electrons $\left(n_{c}\right.$ $=n_{f}=\frac{1}{2}$ ). For these parameters a metal-insulator transition occurs at the critical interaction $U_{c}=2 V$. Fig. 1 shows the double occupation $D(t)$ for different quenches,

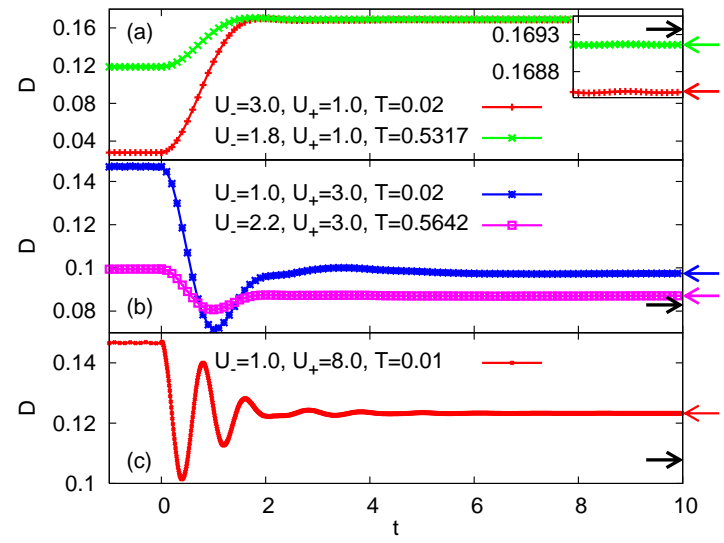

FIG. 1: Double occupation $D(t)$ for quenches to (a) $U_{+}=1$, (b) $U_{+}=3$, and (c) $U_{+}=8$, starting from an initial metallic $\left(U_{-}<2\right)$ or insulating state $\left(U_{-}>2\right)$; the half-bandwidth is $2 V \equiv 2$. In (a) and (b), the internal energy is the same after both quenches. Thick right-pointing arrows mark the double occupation in the thermal state for interaction $U_{+}$with the same density and internal energy. These values differ from the stationary value $D_{\infty}$, marked by left-pointing arrows, which are approached for large times. The inset in (a) shows a magnification of the large- $t$ behavior.

both within and between the two phases. In all cases we observe relaxation to a new stationary value $D_{\infty}$ on the time scale $1 / V$.

The relaxation is almost monotonic when the final interaction $U_{+}$is small (Fig. [1 ), while a distinct overshoot (Fig. 1b) or damped oscillations (Fig. 1F) arise after quenches to large interactions $\left(U_{+}>V\right)$. Such transient oscillations with period $2 \pi / U$ are expected on general grounds when hopping can be neglected [3, 7, 9, 10], because the interaction part of the Hamiltonian alone leads to a strictly $2 \pi / U$ periodic time-evolution operator $\exp \left(-i t U \sum_{i} c_{i}^{\dagger} c_{i} f_{i}^{\dagger} f_{i}\right)$. For small hopping $V \ll U_{+}$ordinary time-dependent perturbation theory then shows that the double occupancy oscillates for times $t \lesssim 1 / V$.

We now discuss the non-thermal character of the final steady state. In case of thermalization it would be fully characterized by a new temperature and a new chemical potential, which are fixed by density and internal energy only. For Fig. 1 the initial temperature is chosen such that the final energy $E(t>0)$ is the same for the two quenches to $U_{+}=V$ (Fig. 1h) and also for the two quenches to $U_{+}=3 \mathrm{~V}$ (Fig. 1 $\mathrm{b}$ ). The stationary value $D_{\infty}$ clearly differs from the double occupation in the thermal state with the same density and internal energy (thick arrows in Fig. 1 a and b). This lack of thermalization is also observed for the occupation $n(\epsilon, t)$ of singleparticle states (Fig. 2), for which the stationary value $n_{\infty}(\epsilon)$ clearly differs from the thermal value with the same $E, n_{c}$, and $U_{+}$. Remarkably, thermalization does not even occur for an infinitesimal interaction quench $\delta U$ $=U_{+}-U_{-} \rightarrow 0$ and infinite waiting time. For this case we find from (9) that $\delta g_{\infty}^{<}(\omega)=-w_{1} \partial_{\omega} r^{<}(\omega) \delta U$. For $T$ 


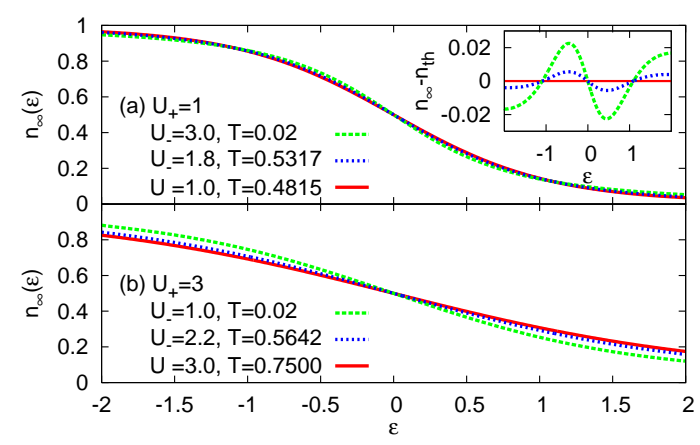

FIG. 2: Stationary $n_{\infty}(\epsilon)$ for quenches to (a) $U_{+}=1$ and (b) $U_{+}=3$ (same as in Fig. 17 and b), compared to the corresponding thermal values (solid red line). The inset shows a magnification of their differences.

$>0$ it can be shown [24] that $g_{\infty}^{<}(\omega)+\delta g_{\infty}^{<}(\omega)$ does not correspond to any thermal state with temperature $T+\delta T$ and chemical potential $\mu+\delta \mu$.

Role of constraints. - Thermalization in the FK model (11) is impossible because the immobile $f$-particles can never find their annealed thermal configuration. In addition the behavior of the $c$-particles is non-ergodic for any fixed configuration $\boldsymbol{n}_{f}=\left\{n_{f, i}\right\}$. This is because for any given $\boldsymbol{n}_{f}$ the Hamiltonian of the $c$ particles is quadratic, say with single-particle eigenstates $\left|\alpha_{\mp}\right\rangle$ and energies $\epsilon_{\alpha_{\mp}}$ before and after the quench. As a consequence the occupation numbers $n_{\alpha_{+}}$after the quench are timeindependent and entirely determined by their equilibrium values before the quench, $n_{\alpha_{+}}=\sum_{\alpha_{-}} f\left(\epsilon_{\alpha_{-}}\right)\left|\left\langle\alpha_{+} \mid \alpha_{-}\right\rangle\right|^{2}$.

Thermalization is prevented by this memory of the initial state that is frozen in $n_{\alpha_{+}}$. Under this assumption the best guess for the steady state of the $c$ particles is a generalized Gibbs ensemble [6], i.e., a density matrix $\rho\left[\boldsymbol{n}_{f}\right]$ which maximizes the entropy $S(\rho)=\operatorname{Tr}(\rho \log \rho)$ subject to all the constraints given for $\left\langle n_{\alpha_{+}}\right\rangle$. Since this $\rho\left[\boldsymbol{n}_{f}\right]$ is a mixture of product states made from $\left\{\left|\alpha_{+}\right\rangle\right\}$, it predicts the site-averaged stationary Green function for a given configuration $\boldsymbol{n}_{f}$ as $g_{\infty}^{<}\left[\boldsymbol{n}_{f}\right](\omega)=2 \pi \sum_{\alpha_{+}} \delta(\omega-$ $\left.\epsilon_{\alpha_{+}}\right) n_{\alpha_{+}}$. This statistical prediction indeed agrees with the exact DMFT result for the infinitesimal interaction quench $\delta U$, as we now show using first-order perturbation theory for $\left|\alpha_{+}\right\rangle$. The first-order energy change is $\delta \epsilon_{\alpha_{-}}=\delta U \sum_{i} n_{f, i}\left\langle\alpha_{-}\left|c_{i}^{\dagger} c_{i}\right| \alpha_{-}\right\rangle$, while the change of $n_{\alpha_{+}}$is of order $\delta U^{2}$. This gives $\delta g_{\infty}^{<}\left[\boldsymbol{n}_{f}\right](\omega)=-2 \pi \partial_{\omega}$ $\sum_{\alpha_{-}} \delta\left(\omega-\epsilon_{\alpha_{-}}\right) f\left(\epsilon_{\alpha_{-}}\right) \delta \epsilon_{\alpha_{-}}=-w_{1} \partial_{\omega} r^{<}\left[\boldsymbol{n}_{f}\right](\omega) \delta U$. Because the probabilities $P\left[\boldsymbol{n}_{f}\right]$ of the $f$ configurations are time-independent and depend only on the initial state of the $c$ electrons, averaging over $\boldsymbol{n}_{f}$ recovers our DMFT result for $\delta g_{\infty}^{<}(\omega)$. Thus generalized Gibbs ensembles provide the appropriate statistical description of this final steady state, at least for simple observables. In this aspect our results, which are strictly valid in infinite dimensions, resemble those for one-dimensional integrable models [6, 7, 8].

Conclusion. - The exact DMFT solution of the FK model after an interaction quench shows that this isolated many-body system relaxes to a new steady state. The momentum occupation and double occupation in the final state do not correspond to any thermal state. Instead these observables are described by means of generalized Gibbs ensembles, averaged over all $f$ configurations.

In general, DMFT has been very successful for correlated systems in equilibrium and gives a good description of local observables in three-dimensional systems. Its application to non-equilibrium phenomena is thus very promising, and DMFT results for quenches in the Hubbard model would be desirable. If the Hubbard model indeed thermalizes, as expected for a non-integrable system [11], this would lead to a crossover between ergodic and non-ergodic regimes. This crossover could be studied experimentally with ultracold atomic gases in optical lattices, e.g., with mixtures of polarized fermionic atoms for which the lattice depth can be tuned separately.

We thank D. Vollhardt, K. Byczuk, and M. Rigol for useful discussions. M.E. acknowledges support by Studienstiftung des Deutschen Volkes. This work was supported in part by the SFB 484 of the DFG.

[1] I. Bloch et al., arXiv:0704.3011

[2] M. Greiner et al., Nature 415, 39 (2002).

[3] M. Greiner et al., Nature 419, 51 (2002).

[4] L. E. Sadler et al., Nature 443, 312 (2006).

[5] T. Kinoshita et al., Nature 440, 900 (2006).

[6] M. Rigol et al., Phys. Rev. Lett. 98, 050405 (2007).

[7] M. Rigol et al., Phys. Rev. A, 74, 053616 (2006).

[8] M. A. Cazalilla, Phys. Rev. Lett. 97, 156403 (2006).

[9] C. Kollath et al., Phys. Rev. Lett. 98, 180601 (2007).

[10] S. R. Manmana et al., Phys. Rev. Lett. 98, 210405 (2007).

[11] M. Rigol, V. Dunjko, and M. Olshanii, arXiv:0708.1324.

[12] W. Metzner and D. Vollhardt, Phys. Rev. Lett. 62, 324 (1989).

[13] A. Georges et al., Rev. Mod. Phys. 68, 13 (1996).

[14] P. Schmidt and H. Monien, arXiv:cond-mat/0202046.

[15] V. Turkowski and J. K. Freericks, Phys. Rev. B 71, 085104 (2005); J. K. Freericks, V. M. Turkowski, and V. Zlatić, Phys. Rev. Lett. 97, 266408 (2006).

[16] K. Byczuk and D. Vollhardt, arXiv:0706.0839.

[17] L. M. Falicov and J. C. Kimball, Phys. Rev. Lett. 22, 997 (1969).

[18] U. Brandt and C. Mielsch, Z. Phys. B 75, 365 (1989).

[19] P. G. J. van Dongen and D. Vollhardt, Phys. Rev. Lett. 65, 1663 (1990); P. G. J. van Dongen, Phys. Rev. B 45, 2267 (1992).

[20] J. K. Freericks and V. Zlatić, Rev. Mod. Phys. 75, 1333 (2003).

[21] F. B. Anders and A. Schiller, Phys. Rev. Lett. 95, 196801 (2005).

[22] Note that adding a time-dependent single-particle potential term $\epsilon(t) N$ at $t=0$ leads to phase factors $\exp \left[i \int_{t}^{t^{\prime}} d \tau\right.$ $\epsilon(\tau)]$ in the Green functions, and thus is of no consequence for equal-time $\left(t=t^{\prime}\right)$ observables.

[23] H. Haug and A.-P. Jauho, Quantum Kinetics in Transport and Optics of Semiconductors (Springer, Berlin, 1996).

[24] M. Eckstein et al., unpublished. 